\title{
Long-Term Modulation of the Galactic Cosmic-Ray Fluctuation Spectrum
}

\author{
S. A. Starodubtsev ${ }^{1 *}$ and I. G. Usoskin ${ }^{2}$ \\ ${ }^{1}$ Institute of Cosmophysical Research and Aeronomy, Siberian Branch, Russian Academy of Sciences, \\ pr. Lenina 31, Yakutsk, 677891 Russia \\ ${ }^{2}$ Sodankylä Geophysical Observatory (Oulu Unit), University of Oulu, P.O. Box 3000, FIN-90014, Finland
}

Received February 6, 2003

\begin{abstract}
We study the temporal behavior of the power spectra for Galactic cosmic-ray fluctuations during the last two solar cycles. We use the 5-min data for 1980-2002 corrected for the barometric effect from two widely separated high-latitude cosmic-ray stations, Tixie Bay and Oulu. The cosmicray fluctuation spectrum is shown to be subjected to a regular long-term modulation with a period of about 11 years in phase with the solar cycle, in accordance with the variations in the inertial part of the turbulence spectrum for the interplanetary magnetic field. Based on independent measurements, we confirm the previously detected cosmic-ray fluctuation power enhancement at the maximum of the 11-year solar cycle and its subsequent decrease at minimum solar activity using new, more extensive data sets. We reach the conclusion about the establishment of a new cosmic-ray modulation phenomenon that has not been described previously in scientific literature. (C) 2003 MAIK "Nauka/Interperiodica".
\end{abstract}

Key words: cosmic rays, solar activity.

\section{INTRODUCTION}

Of the entire observed variety of cosmic-ray (CR) variations-temporal and spatial Galactic CR flux variations - the class of short-period variationswith periods from several minutes to several hoursis least understood. In scientific literature, this class of variations is called CR fluctuations. This name in many respects stems from the fact that the fluctuation amplitude is comparable to the noise level recorded by ground-based CR detectors. In general, a groundbased study of the CR fluctuations is based on neutron-monitor data due to the high efficiency of the counters and the large area of the detectors. In a 5-min recording mode, the statistical measurement error of the Galactic CR intensity for various stations is $\sigma \approx 0.7 \%$, while the amplitude of the fluctuations themselves rarely exceeds $1.0 \%$. Thus, the main problem in studying the CR fluctuations is to isolate the useful signal against a high noise background. The main tool for solving this problem is a spectral analysis of time series.

A systematic study of the physical nature of the CR fluctuations and the dynamics of their power spectrum began 30 years ago (Kozlov et al. 1973). By now, undeniable progress has been made in this field of studying the CR variations. Analysis of

${ }^{*}$ E-mail: starodub@ikfia.ysn.ru data from the numerous experiments carried out at different CR stations in different countries established the interplanetary origin of the CR fluctuations and revealed the characteristic frequency dynamics of the fluctuation power spectra before the arrival of large-scale solar-wind (SW) disturbances at the Earth's orbit (Starodubtsev 1985; Vashenyuk et al. 1995; Dorman et al. 1995; Perez-Peraza et al. 1998; Kudela et al. 1999; Kozlov et al. 2001) and their relationship to the IMF turbulence spectrum (Owens 1974; Toptygin 1983; Berezhko and Starodubtsev 1988).

The CR fluctuation spectra are very dynamic; they continuously exhibit variations both in the frequency range and in power, depending on the SW state. As a result, the information flow contained in them is very large. Therefore, analysis of the spectra, especially on long time scales, encounters considerable difficulties. To overcome these difficulties, some authors introduce various CR fluctuation indices that describe the temporal behavior of the CR power spectra (Kudela and Langer 1995; Kudela et al. 1999; Kozlov et al. 2001). These indices allow us to study the dynamics of the spectra on long time scales and to draw definitive conclusions.

Such an approach was used by Berezhko et al. (1993). Based on 5-min data from the Tixie neutron monitor, they calculated the monthly mean index of the CR fluctuation spectral power density $\bar{G}$ by using 
Basic parameters of the Tixie and Oulu high-latitude CR stations

\begin{tabular}{c|c|c|c|c}
\hline Station & Device type & $\begin{array}{c}\text { Geographic } \\
\text { latitude } \lambda\end{array}$ & Geographic longitude $\phi$ & $\begin{array}{c}\text { Geomagnetic rigidity } \\
\text { cutoff threshold } R_{C}, \mathrm{GV}\end{array}$ \\
\hline Oulu & $9 \mathrm{NM} 64$ & 65.02 & 25.2 & 0.81 \\
Tixie & 12NM64 & 71.60 & 128.9 & 0.53 \\
\hline
\end{tabular}

the fast Fourier transform and studied its behavior during the solar cycle (1980-1990). As a result of their studies, they concluded that there was a significant correlation between the CR fluctuation amplitude and the level of solar activity and hypothesized the corresponding spectral evolution of the magnetosonic SW turbulence associated with solar-flare activity.

If this modulation effect in Galactic CRs actually exists, then it must also be observed in data from other stations with parameters similar to those of the Tixie CR station. Here, our goal is to reliably establish this phenomenon by using independent measurements at two CR stations on a long time scale.

\section{DATA AND THE TECHNIQUE OF ANALYSIS}

In this paper, we use continuous 5-min CR recording data from the $\mathrm{CR}$ stations Tixie (Russia) and Oulu (Finland). The Tixie and Oulu data cover the periods from January 10, 1980, through June 25, 1991, and from January 19, 1985, through October 16,2002 , respectively. Thus, the observational data mutually overlap by 6.5 years and make it possible to mutually calibrate the measurements. As a result, the period under study spans more than 22 years (from 1980 until 2002), begins from the maximum of solar cycle 21 , and includes the maximum of solar cycle 23.

The table lists the basic parameters of the stations. The two high-latitude stations are located at the sea level and have similar geomagnetic rigidity cutoff thresholds $R_{C}<1 \mathrm{GV}$; i.e., the effect of the Earth's magnetic field on the recorded CR particle fluxes is weak. Thus, the minimum energies of the CR particles that arrive at the stations are mainly determined by the thickness of the atmosphere. Both stations have narrow asymptotic acceptance cones for CR particles with energy up to $10 \mathrm{GeV}$ (Inoue et al. 1983). The stations are widely separated in longitude, by more than $100^{\circ}$, suggesting an independence of the time series used in our analysis. Both stations are equipped with similar instruments-NM-64 neutron monitors, but a different number of counters: 12 at the Tixie station and 9 in Oulu, which affects the statistical counting of the instruments.
To avoid erroneous conclusions, in the primary reduction of the $\mathrm{CR}$ measurements great attention should be paid to the corrections for meteorological effects. Since the effect of the Earth's atmospheric temperature on the neutron CR component is less than $0.01 \%$ (Dorman 1974), we analyze here 5-min data corrected only for the much stronger barometric effect.

An indispensable condition for using data in analysis is their quality. As the initial data (as in Berezhko et al. 1993), we took a daily realization (288 points). In the absence of apparent errors and gaps for more than two hours ( 24 points), we considered them suitable for our analysis. In addition, we excluded from our analysis the periods when solar CR events were recorded at the stations; in general, such solar proton events have a rapid $(\sim 1 \mathrm{~h})$, sharp rise and a large amplitude, which significantly distorts the results of the spectral analysis. We did not exclude Forbush decreases from our analysis, because the CR decrease in the 5-min data has the pattern of a low-frequency trend, which can be eliminated by digital filtering.

To compare the data from the two different stations, we expressed the CR intensity in percents of the average level for each data realization.

The first step in preparing the time series for spectral analysis is the standard procedure of reducing the data to a zero mean. The main problem in the spectral analysis is the signal-to-noise ratio. To obtain correct estimates of the calculated quantities, it is necessary to avoid here the power redistribution in the spectra in frequency.

Therefore, the next step-the reduction of the data to a quasi-stationary form - is achieved by highfrequency filtering. It can be implemented by any digital filter with an appropriate transfer function. An important condition at this step is the choice of a working filter frequency band $\nu_{1}<\Delta \nu<\nu_{2}$. In our case, this band is determined at high frequencies by the data-sampling step $\Delta t=300 \mathrm{~s}$, which corresponds to the Nyquist frequency $\nu_{2}=1.67 \times 10^{-3} \mathrm{~Hz}$. The low frequency $\nu_{1}$ of the working filter-frequency band $\Delta \nu$ is more difficult to choose, because it depends in many respects on the physical nature of the CR fluctuations under study. Here, taking into account the results of our previous studies (Berezhko and 
Starodubtsev 1988; Berezhko et al. 1993; Starodubtsev 1999), we again chose it to be $\nu_{1}=1.67 \times$ $10^{-4} \mathrm{~Hz}$.

In the third step of our analysis, we calculated the daily CR fluctuation power spectra by using the standard algorithm of Blackman and Tukey (1958). To reduce the power leakage through sidelobes, we used the Tukey correlation window, with the number of degrees of freedom being 12. Subsequently, from the daily spectra obtained in this way, we calculated the 27 -day-averaged spectra $P(\nu)$ by using the ensemble averaging procedure; the errors in the estimated CR fluctuation power decreased sharply, while the number of degrees of freedom increased to 324 .

It should be noted that Berezhko et al. (1993) used a different method of spectral analysis, a fast Fourier transform. Apart from its advantage- a considerable reduction in the computational time (by a factor of $\sim 100$ ) - this method has a serious drawback: when the spectrum is calculated from one data realization, the number of degrees of freedom is 2 ; therefore, the errors in the estimated spectral power are large. To overcome this effect, we used the procedure for ensemble averaging of the spectra, which increases the number of degrees of freedom and appreciably reduces the confidence intervals in estimating the power.

Thus, using the Blackman-Tukey method, we can not only check the previous results but also improve them.

In contrast to the monthly average, the 27-day average, which is related to the synodic solar rotation period, has a physical meaning and allows us to get rid of the quite possible effects of longitudinal inhomogeneities in the SW and the solar corona.

Next, to study the temporal behavior of the CR fluctuation power, we calculated the CR fluctuation power index $P 2$ in the frequency range under investigation from the 27-day-averaged spectra (Kudela and Langer 1995):

$$
P 2=\int_{\nu_{1}}^{\nu_{2}} P(\nu) d \nu .
$$

In contrast to the $\bar{G}$ index (Berezhko et al. 1993), this quantity is the CR fluctuation power in the chosen frequency range, and we used it as the CR fluctuation index here.

In our analysis, we also used the 27-day data on the CR intensity $J$ acquired at the Oulu station and normalized to September 1985. To avoid possible errors like a long-term trend, we corrected the data according to the information at http://cosmicrays.oulu.fi /readme.html\#norm.
As the solar-activity index, we used the 27-day data on the sunspot number $R_{Z}$ from the OMNI database at http://nssdc.gsfc.nasa.gov/omniweb/ ow.html.

All of the data were time referenced according to the Bartels rotation numbers.

\section{RESULTS AND DISCUSSION}

Figure 1 shows the sunspot number $R_{Z}$ (Fig. 1a), which is the universally accepted solar-activity index, the CR intensity $J$ recorded at the Oulu station (Fig. 1b), and the CR fluctuation indices P2 (Fig. 1c) calculated for the two CR stations for the period under study. For the Tixie and Oulu stations, the $P 2$ index is indicated by filled squares and open circles, respectively. To mutually normalize the data, the CR fluctuation index $P 2$ was multiplied by a constant factor of 1.33 , because of the different statistical counting of the neutron monitors at these stations.

We clearly see from Figs. $1 \mathrm{a}$ and $1 \mathrm{~b}$ that the sunspot numbers and the CR intensity are in antiphase, whereas the CR fluctuation indices (Fig. 1c), on the contrary, vary synchronously with the solarcycle phase. The CR fluctuation indices P2 calculated from the data of the two stations closely match in the overlapping time interval and complement each other, which allows them to be considered as a single index throughout the interval under study.

Our calculation of the correlation matrix between the CR index $P 2$ for the Oulu station and the sunspot number $R_{Z}$ for Bartels rotations 2070-2299 suggests a significant correlation $\rho(\tau)=0.67$ for a zero shift and its increase to $\rho=0.71$ for a shift of $\tau=10$ solar rotations. This implies that our result is with a high probability (a significance level $\alpha<0.1$ ), not accidental and that the $P 2$ index peaks at the beginning of the decline in solar activity, when enhanced flare and coronal solar activity is observed (Starodubtsev 1999). The derived correlation coefficients for the Oulu station closely match those calculated previously by Berezhko et al. (1993) for the Tixie station $(\rho(0)=0.60, \rho(10)=0.78, \alpha<0.05)$. The small discrepancy stems from the fact that here we use the 27-day CR and solar-activity indices rather than the monthly indices, as in the cited paper. In addition, the $P 2$ and $\bar{G}$ indices have a slightly different physical meaning, and they were calculated by using different techniques.

It also follows from Figs. $1 \mathrm{~b}$ and $1 \mathrm{c}$ that the CR fluctuation index anticorrelates with the CR intensity. In this case, the maximum value of the correlation function $\rho(\tau)=-0.78$ is observed without any shift $(\tau=0)$. Thus, the maximum CR fluctuation amplitude is observed when the CR intensity $J$ reaches its minimum at high solar activity. 

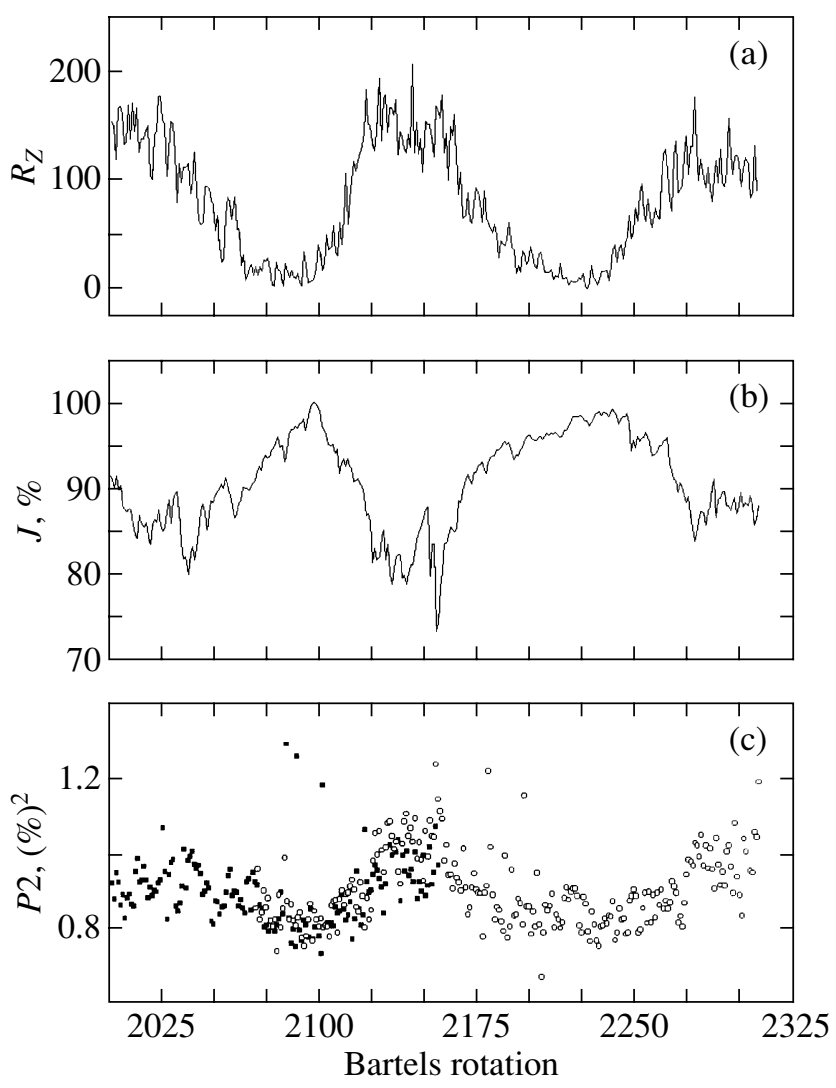

Fig. 1. The temporal behavior of the sunspot number $R_{Z}$ (a), the CR intensity recorded at the Oulu station $J$ (b), and the CR fluctuation index $P 2$ (c) relative to the Bartels rotation number.

At the same time, the CR intensity itself is in antiphase with the sunspot number, and the maximum of the correlation function $\rho(\tau)=-0.90$ is observed for a shift of $\tau=5$ Bartels rotations, which corresponds to the mean delay between the solaractivity and CR variations (Usoskin et al. 1998). It thus follows that the ground-level CR intensity minimum slightly lags behind the solar maximum, and the intensity variations themselves are also related to manifestations of flare and coronal solar activity.

Let us briefly discuss the possible physical mechanism of the observed CR fluctuations. Several authors (Owens 1974; Toptygin 1983; Berezhko and Starodubtsev 1988) developed a theory of CR fluctuations in random magnetic fields and compared it with observations. In these papers, the fact that a modulation can arise from both Alfvén and magnetosonic waves during the interaction of high-energy CRs $(E \sim 1 \mathrm{GeV})$ with the interplanetary magnetic field engages our attention. In the former and latter cases, the anisotropic and much larger (in magnitude) isotropic parts of the CR distribution function are modulated, respectively. In the case of Alfvén waves, the observed CR fluctuation spectra are satisfactorily described by the theory down to frequencies $\nu<10^{-4} \mathrm{~Hz}$ (Owens 1974). This restriction can be understood if we take into account the fact that, in this case, a CR modulation occurs during the resonant interaction with the random IMF component; for typical SW conditions (a magnetic field intensity $B=6 \mathrm{nT}$, a SW speed $U=400 \mathrm{~km} \mathrm{~s}^{-1}$ ), the resonant frequencies for Alfvén waves are $\nu_{\text {res }} \approx 1.18 \times$ $10^{-3} \mathrm{~Hz}$ and $\nu_{\text {res }} \approx 1.05 \times 10^{-4} \mathrm{~Hz}$ for CR particles with energies of 5 and $500 \mathrm{MeV}$, respectively. At the same time, the CRs recorded by neutron monitors, even at high latitudes, have a minimum energy $E>500 \mathrm{MeV}$. In contrast, for the CR modulation by fast magnetosonic SW turbulence, the theoretical CR fluctuation spectra in the frequency range studied here agree well with the observed spectra (Berezhko and Starodubtsev 1988).

Thus, the results presented in the figure are consistent with the conclusions of Berezhko et al. (1993) and also lead us to conclude that the small-scale $\left(\sim 10^{10}-10^{12} \mathrm{~cm}\right)$, presumably magnetosonic SW turbulence level must behave in a similar way during the solar cycle. Indeed, as we showed previously (Starodubtsev 1999), significant (by more than two orders of magnitude) regular variations are also observed during the 11-year solar cycle in the inertial 
part of the IMF turbulence spectrum in the same frequency range. The local (near the Earth's orbit) generation of MHD waves by the streams of lowenergy CRs $\left(E_{p} \sim(0.01-10) \mathrm{MeV}\right)$ associated with coronal solar activity (flares and coronal mass ejections) may be responsible for this phenomenon (see Starodubtsev et al. (1999) and references therein).

Unfortunately, the available 5-min IMP-8 IMF data only slightly (about 7 years) overlap with the $\mathrm{CR}$ data from the two stations. Therefore, we cannot quantitatively study the correlation between the CR fluctuation index $P 2$ and the SW turbulence level during the solar cycle. In addition, the IMF measurements are discrete in nature and carry information about the local properties of the medium, while CRs carry information about the integrated properties, and various types of MHD waves are simultaneously present in the observed IMF spectra. Therefore, a high, significant correlation between them on large time scales can hardly be expected.

\section{CONCLUSIONS}

Our results provide evidence for the long-term high-frequency modulation of the Galactic CR fluctuation spectrum. In essence, we have confirmed a new, previously unknown CR phenomenon, which was first described by Berezhko et al. (1993). It is also confirmed by the corresponding measurements of the inertial part of the IMF fluctuation spectrum during the solar cycle (Starodubtsev 1999), which would be expected from the nature of the CR fluctuations (Berezhko and Starodubtsev 1988).

This phenomenon is observed for particles with energy $E$ of at least $\sim 1 \mathrm{GeV}$. In the future, it will be undoubtedly of interest to determine the energy thresholds of the high-frequency CR flux modulation. This requires data from mid- and low-latitude CR stations with a sampling step of several minutes in a time interval that covers at least one solar cycle, as well as low-energy CR flux measurements in interplanetary space from spacecraft.

One might expect a considerable increase in the IMF turbulence level in 2003 from the behavior of the CR fluctuation index $P 2$, which can appreciably affect the behavior of many parameters of the interplanetary medium and the Earth's magnetosphere.

At present, the 5-min data from the neutron monitors of the above stations are available online in the Internet at http://cosmicrays.oulu.fi and http://www.ysn.ru/ipm.

\section{ACKNOWLEDGMENTS}

We are grateful to the National Space Science Data Center, the Goddard Space Flight Center (Greenbelt, MD, USA), for the OMNI database. This work was supported in part by the Russian Foundation for Basic Research (project no. 01-02-17278) and the INTAS (grant no. 2000-0752).

\section{REFERENCES}

1. E. G. Berezhko, I. A. Brevnova, and S. A. Starodubtsev, Pis'ma Astron. Zh. 19, 749 (1993) [Astron. Lett. 19, 304 (1993)].

2. E. G. Berezhko and S. A. Starodubtsev, Izv. Akad. Nauk SSSR, Ser. Fiz. 52, 2361 (1988).

3. R. B. Blackman and J. W. Tukey, The Measurement of Power Spectra (Dover. Mineola, New York, 1958).

4. L. I. Dorman, Cosmic Ray Variations and Space Explorations (North-Holland, Amsterdam, 1974), p. 675.

5. L. I. Dorman, G. Villoresi, A. V. Belov, et al., Nucl. Phys. B (Proc. Suppl.) 39, 136 (1995).

6. A. Inoue, M. Wada, and I. Kondo, Cosmic Ray Tables (Institute of Physical and Chemical Research, Itabashi, Tokyo, 1983), No. 1.

7. V. I. Kozlov, A. I. Kuzmin, G. F. Krymsky, et al., Proc. of the 13th ICRC, Denver, USA 2, 939 (1973).

8. V. I. Kozlov, S. A. Starodubtsev, V. G. Grigor'ev, et al., Izv. Ross. Akad. Nauk, Ser. Fiz. 65, 385 (2001).

9. K. Kudela and R. Langer, Contrib. Astron. Obs. Skalnate Pleso 25, 5 (1995).

10. K. Kudela, I. M. Martin, and P. Bobik, Proc. 26th ICRC, Salt Lake City, Utah, USA 6, 444 (1999).

11. K. Kudela, M. Storini, M. Hofer, and A. Belov, Space Sci. Rev. 93, 153 (2000).

12. A. J. Owens, J. Geophys. Res. 79, 895 (1974).

13. J. Perez-Peraza, A. Leyva-Contreras, I. Ya. Libin, et al., Geofis. Int. 37, 87 (1998).

14. S. A. Starodubtsev, Geomagn. Aéronom. 25, 997 (1985).

15. S. A. Starodubtsev, Pis'ma Astron. Zh. 25, 626 (1999) [Astron. Lett. 25, 540 (1999)].

16. I. N. Toptygin, Cosmic Rays in Intraplanetary Magnetic Fields (Nauka, Moscow, 1983).

17. I. G. Usoskin, H. Kananen, G. A. Kovaltsov, et al., J. Geophys. Res. 103, 9567 (1998).

18. E. V. Vashenyuk, V. S. Smirnov, V. S. Ismagilov, et al., Proc. of the 24th ICRC, Roma, Italy 4, 880 (1995).

Translated by G. Rudnitskii 